\title{
Acute Paraplegia Secondary to Thoracic Disc Herniation of the Adjacent Segment Following Thoracolumbar Fusion and Instrumentation
}

\author{
Mohammad Ibrahim Badra ${ }^{1}$, Richard Assaker ${ }^{2}$, Ramzi Sharif Musharrafieh ${ }^{1}$ \\ ${ }^{I}$ Department of Orthopedic Surgery, Clemenceau Medical Center, Beirut, Lebanon \\ ${ }^{2}$ Department of Neurosurgery, Lille University Hospital, Lille, France
}

\begin{abstract}
Proximal junctional disease is a well-recognized postoperative phenomenon in adults who are undergoing long thoracolumbar fusion and instrumentation, and is attributed to increased a junctional stress concentration. In general, the onset of symptoms in these patients is insidious and the disease progresses slowly. We report on a contrary case of rapidly progressing paraplegia secondary to acute disc herniation at the proximal adjacent segment after long posterior thoracolumbar fusion with cement augmentation at the upper instrumented vertebra and the supra-adjacent vertebra. The patient was treated with a discectomy through the costotransverse approach combined with extension of the posterior instrumentation. The patient's neurological status improved markedly. Stress concentration at the proximal junction disc space may have caused accelerated disc degeneration which in turn lead to this complication.
\end{abstract}

Keywords: Thoracic disc herniation; Adjacent segment disease; Instrumented thoracolumbar fusion; Paraplegia

\section{Introduction}

Adult thoracolumbar fusion and instrumentation is a relatively common surgical procedure that is used for treatment of different spine pathologies including degenerative scoliosis, flat-back deformities and revision cases where extension of instrumentation to the upper thoracic segments is often needed to achieve an acceptable sagittal balance and to decrease the risk of construct failure. Several adjacent segment problems, mainly at the proximal level, have been reported after such procedures [1-6]. These problems include proximal adjacent disc degeneration, failure of the instrumentation at the upper-instrumented vertebra (UIV) and fracture of the UIV or the first mobile vertebra above it, leading to sagittal thoracic decompensation. Vertebral body augmentation with polymethylmethacrylate (PMMA) has been suggested as a means of enhancing the pullout strength of the screws at the UIV and reducing the supra-adjacent vertebral fractures $[7,8]$, thus decreasing the risks for adjacent segment diseases.

Proximal junctional severe disc degeneration causing spinal stenosis has also been reported as a complication [3]. However, to our knowledge, only one case of thoracic disc herniation of the adjacent segments following long instrumented thoracolumbar fusion with rapidly progressing myelopathy has been described [4]. We report on a case of acute upper thoracic disc herniation (T4-T5) with rapidly progressing paraplegia after T5 to S1 spinal fusion and in-

Received Sep 14, 2011; Revised Oct 18, 2011; Accepted Oct 26, 2011

Corresponding author: Mohammad Ibrahim Badra

Department of Orthopedics Surgery, Clemenceau Medical Center, PO Box 11-2555-Beirut, Lebanon

Tel: +961-3-020322, Fax: +961-1-364464, E-mail: mohammad.badra@cmc.com.lb 
strumentation with bone cement augmentation at the adjacent segments.

\section{Case Report}

A 57-year-old obese female patient who presented initially for evaluation of severe mid-back pain and an inability to stand up straight. One year prior to her presentation, she had had an instrumented anterior and posterior spine fusion from $\mathrm{T} 11$ to $\mathrm{S} 1$ for a degenerative lumbar disc disease. Neurological examination showed intact motor power with normal sensation in both upper and lower extremities. A standing anteroposterior (AP) and lateral X-rays of the dorso-lumbar spine showed segmental pedicular screw instrumentation with interbody cages done at every level from T11 to S1 (Fig. 1). There was an apparent sagittal spine malalignment with a complete loss of lumbar lordosis and positive sagittal vertical axis. The uppermost pedicular screws at T11 level appeared to be backed out. There was no fracture of the upper instrumented vertebra or the supra-adjacent vertebra.

After thorough discussion of the treatment options with the patient, she elected to have a trial of conservative management. She was started on a physical therapy program to strengthen her back musculatures along with analgesics and anti-inflammatory medications for pain. Follow-up after 6

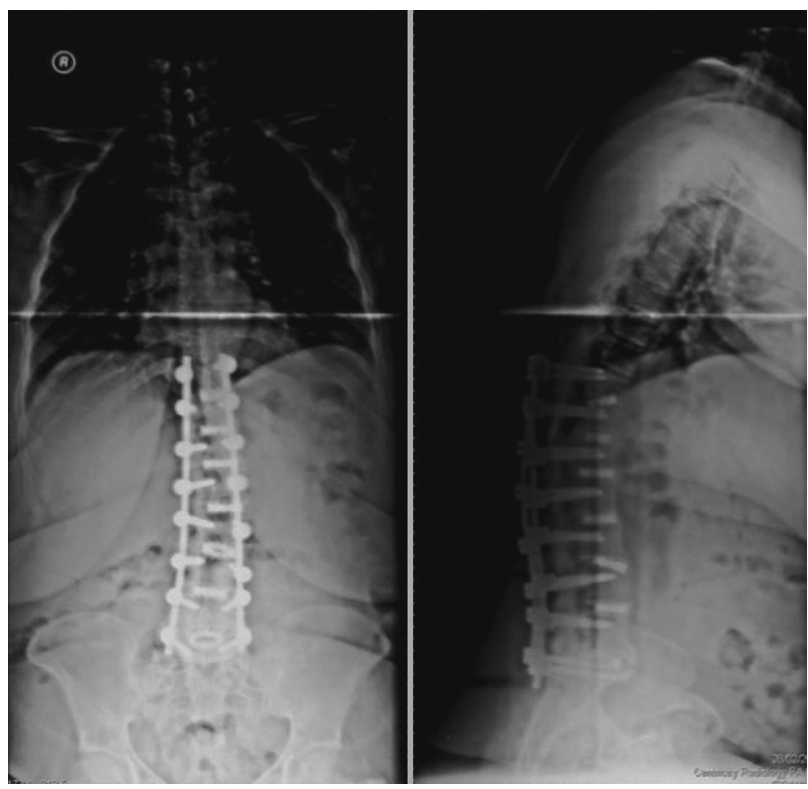

Fig. 1. Standing anteroposterior and lateral X-rays of the dorso-lumbar spine showing a failure of the pedicular screws at T11. Note the iatrogenic flat-back deformity with loss of sagittal spine alignment and +ve sagittal vertical axis. months showed minimal response with persistence of symptoms, mainly back pain and poor standing posture. She underwent extension of the posterior spine fusion and instrumentation up to T5 with augmentation of the pedicle screws at T5 with PMMA cement and prophylactic vertebroplasty at T4 (Fig. 2). Intraoperatively, care was taken not to disrupt the interspinous or the supraspinous ligament between T4 and T5 in order to avoid adjacent segment problems, particularly proximal junctional kyphosis.

Postoperatively, the patient progressed satisfactorily. She was able to stand up straight with only tolerable back pain. However, two months after surgery, she developed sudden motor and sensory impairment of both lower extremities while getting out of bed. She said there was no history of trauma or of falling. She was taken to a nearby hospital where physical exam showed complete loss of motor power and sensation in both lower extremities. Computed tomography (CT)-myelogram done at that hospital showed signs of obstruction at T4-T5 level. She was transferred to our hospital four days later. Neurological examination confirmed the absence of motor power in all muscle groups with absent sensation in all dermatomes of both lower extremities (ASIA A). The last normal sensory level was T4 (breast level). She had positive bilateral Babinski reflexes with loss of bowel and bladder continence. X-rays of the dorsal spine showed

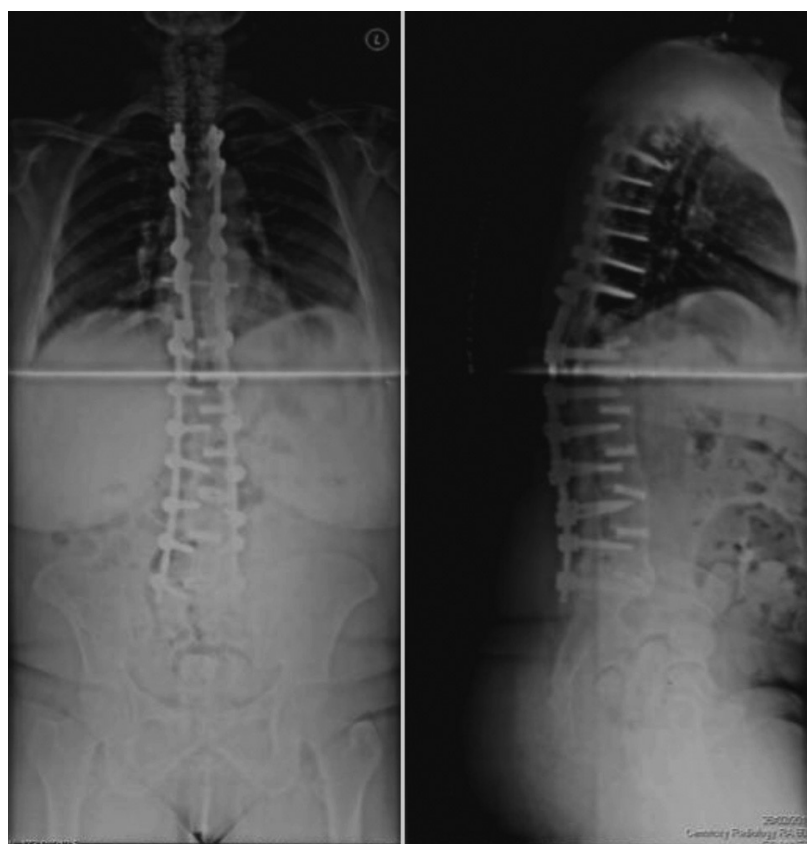

Fig. 2. Postoperative anteroposterior and lateral plain radiographs of the dorso-lumbar spine showing an extension of the instrumentation up to $T 5$ vertebra with cement augmentation of the pedicular screws at T5 level and prophylactic vertebroplasty at T4 level. 
no hardware failure or any vertebral collapse at the proximal junctional area (Fig. 3). Reviewing the CT-myelogram confirmed the obstruction of dye flow at T4-5 level. She was taken immediately to the operating room where posterior spine decompression through laminectomies was done from T3 to T5 with extension of the instrumentation to T3 using transverse process hooks.

On the second day, the patient showed progressive improvement in her neurological status. She regained normal sensation in both lower extremities and started to wiggle her toes. Repeated CT scanning showed no hardware failure or

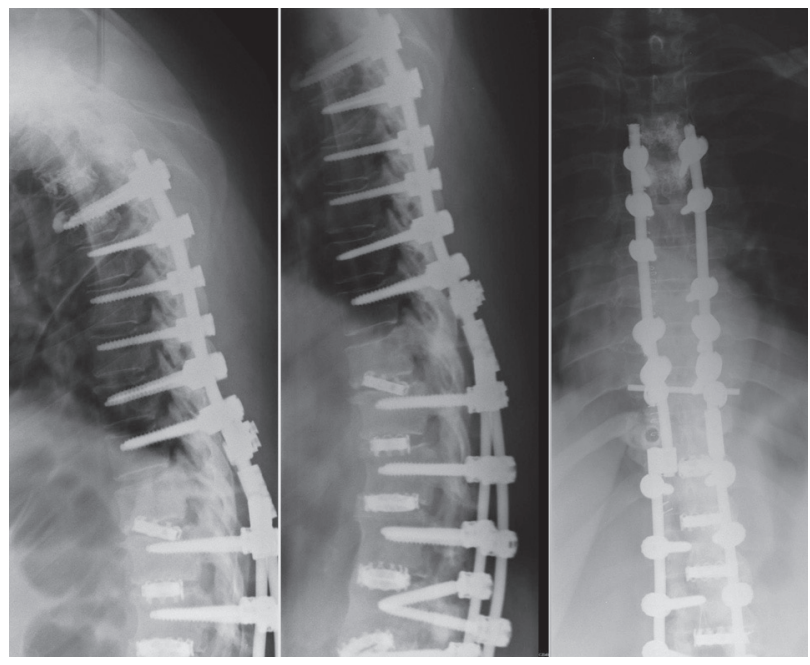

Fig. 3. Plain radiographs of the dorso-lumbar spine after the onset of symptoms showing no hardware failure or vertebral body fracture at the proximal junctional area. any vertebral collapse of either T4 or T5 (Fig. 4). MRI suggested T4-T5 acute disc herniation compressing the spinal cord (Fig. 5).

The patient then underwent T4-T5 discectomy done through a costo-transverse approach along with interbody fusion at the same level using autologous bone graft and extension of the instrumentation up to T2 using pedicular

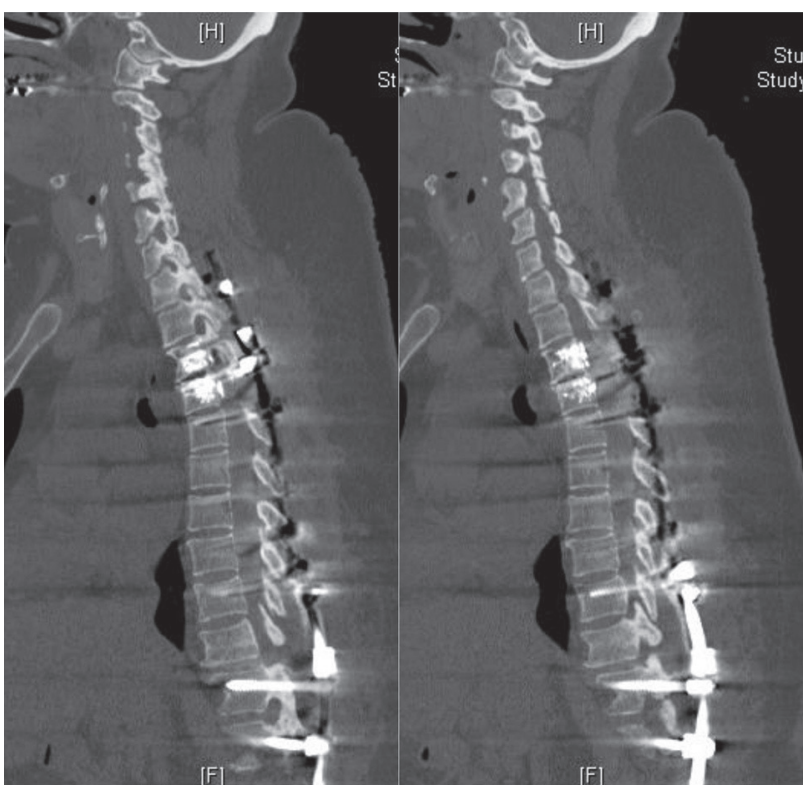

Fig. 4. Computed tomography scan of the dorso-lumbar spine showing no fracture of either T4 or T5 vertebral bodies with no hardware failure at any level. Note the presence of a gas shadow at the T4-T5 intervertebral disc space suggestive of a vacuum phenomenon at this level.

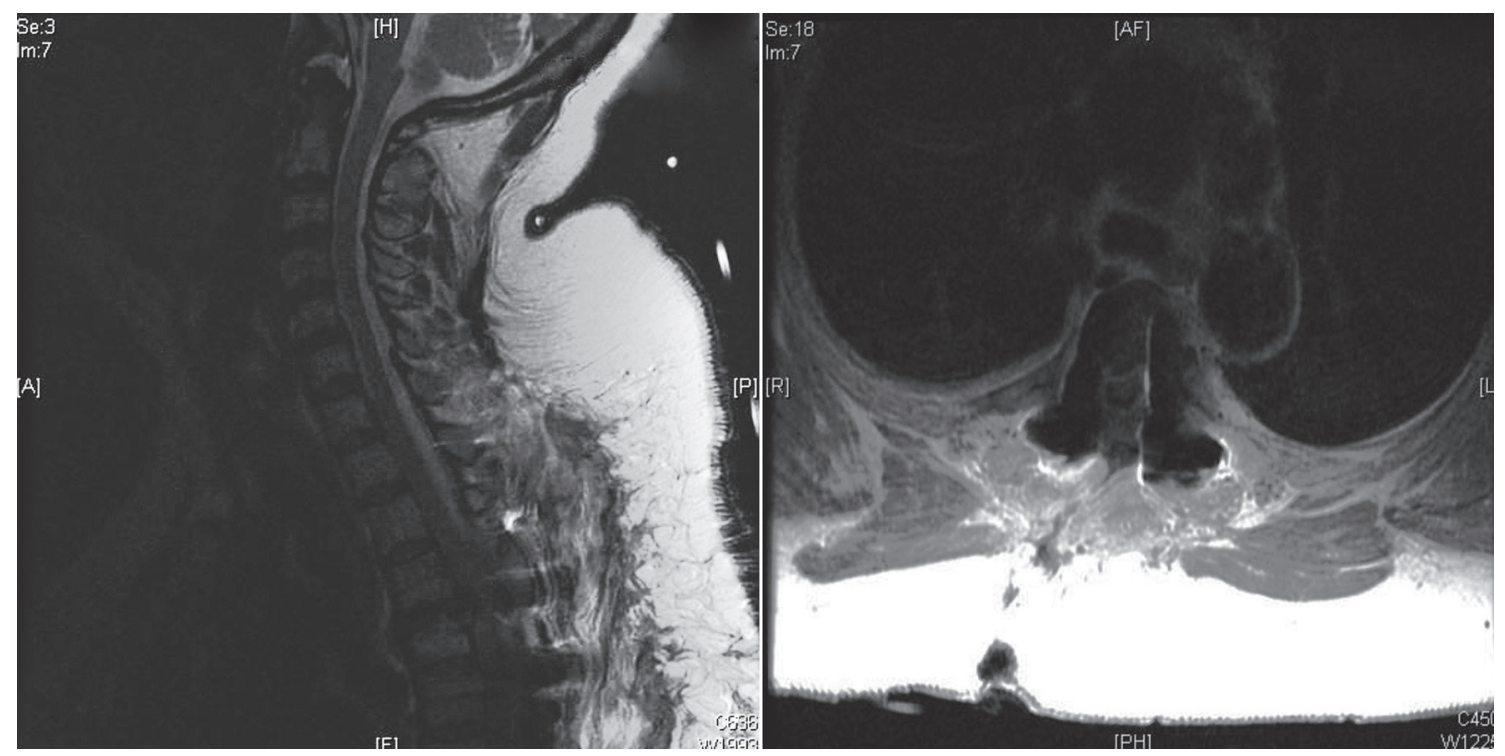

Fig. 5. Sagittal and axial T2-weighted magnetic resonance images demonstrating acute disc herniation at T4-T5 level compressing the spinal cord. 
screws.

Postoperatively, the patient's neurological status continued to improve gradually. At two weeks after operation, she had normal sensation in both her lower extremities with good bowel and bladder control. She was able to move her feet and adduct her legs with some quadriceps activities. She was started on an extensive physical therapy program and she is showing a remarkable improvement in her motor power.

\section{Discussion}

Adjacent segment disease following long instrumented thoracolumbar fusion has been widely reported in medical literature [1-3].

Stress concentration on the proximal junctional area after such procedures may lead to several junctional problems such as proximal adjacent disc degeneration, stress fracture of the upper instrumented vertebra or the first mobile vertebra above it with a loss of sagittal balance [4-6]. In a retrospective study that included 35 patients with long lumbar-instrumented fusion, Kim et al. [9] reported a $43 \%$ prevalence of proximal adjacent segmental problems. In another retrospective study, Kim et al. [10] reported a $23 \%$ (23/99 patients) incidence rate of sagittal thoracic decompensation after long lumbar spinal instrumentation and fusion. The causes of sagittal thoracic kyphosis in this study comprised 14 of acute angular kyphosis caused either by severe proximal disc degeneration or compression fracture at the UIV and 9 cases of long sweeping multisegemental kyphosis secondary to a more global progression of the kyphosis. Sagittal thoracic decompensation secondary to compression fractures at the UIV occurred at an average of 2.7 years postoperatively compared to 4.2 years in patients with sagittal decompensation secondary to severe proximal disc degeneration.

Watanabe et al. [5] described 2 types of proximal junctional fracture after long instrumented fusion in adults. The first type involved a collapse of the UIV followed by subluxation of the supra-adjacent vertebra. This type of fracture tends to occur soon after surgery (average, 2.8 months) and may be provoked by marked correction of the sagittal malalignment with rigid segmental pedicle screw fixation that results in a concentration of mechanical stress on the uppermost-instrumented vertebra. The second type involves fracture of the supra-adjacent vertebra without any collapse of the UIV. This type of fracture tends to occur after a long interval (average, 33.2 months) and might be caused by stress concentration on the unfused adjacent segments above fixed spinal segments or may alternatively represent an occasional fracture normally seen in these patients according to the natural history of the disease's progression.

DeWald et al. [11] examined elderly patients with osteoporotic bones who had multisegmental fusion for spinal deformity. $26 \%$ developed a compression fracture of either the last instrumented vertebra or the vertebra above it.

Bone cement augmentation of the pedicular screws at the UIV with prophylactic vertebroplasty of the supra-adjacent vertebra has been suggested as a way of preventing failure after long instrumented fusion in adults [7,12-14]. Voros et al. [14] presented a series of 11 patients with prophylactic vertebroplasty at the UIV and the vertebra above it after long instrumented fusion and found no significant change in kyphosis over a follow-up period of 2 years. Lattig [13] reported on 6 patients treated using a similar technique and also found no loss of correction, fracture or screw loosening at the proximal adjacent levels after a one year follow-up.

Proximal adjacent disc degeneration after long instrumented spinal fusion has been widely reported upon $[2,3,9]$. However, most of the cases are either asymptomatic or associated with back pain and rarely with neurological abnormality. Oh et al. [4] described a T9-T10 thoracic disc herniation at the proximal adjacent segment with acutely progressing myelopathy 3 years after T10 to S1 posterior spinal fusion and instrumentation. In our case, however, there was no failure of the augmented UIV or the supra-adjacent vertebra; however, accelerated disc degeneration with acute disc herniation occurred approximately 8 weeks postoperatively. We believe that stress concentration at the proximal adjacent disc space between two stiff vertebrae led to acute failure of the disc resulting in rapidly progressing myelopathy.

To decrease the risk of such complication, practitioners should make sure that the disc above the UIV is a healthy one and preserve as much as possible the supraspinous and interspinous ligaments as well as the facet joints between the UIV and the supraadjacent vertebra.

We present an unusual case of complete paraplegia secondary to acute thoracic disc herniation at the proximal junction after along instrumented fusion with cement augmentation at the UIV and the supra-adjacent vertebra. Failure at the proximal junction after such procedures has been attributed to either vertebral bone fracture or hardware loosening but rarely to the disc itself. We report on this case to highlight the fact that failure at the proximal junction 
after long instrumented fusion can happen at the level of the disc itself, and may lead to catastrophic neurological complication.

\section{Conflict of interest}

No potential conflict of interest relevant to this article was reported.

\section{References}

1. Cheh G, Bridwell KH, Lenke LG, et al. Adjacent segment disease followinglumbar/thoracolumbar fusion with pedicle screw instrumentation: a minimum 5-year follow-up. Spine (Phila Pa 1976) 2007;32:2253-7.

2. Kim KS, Hwang HS, Jeong JH, Moon SM, Choi SK, Kim SM. The change of adjacent segment and sagittal balance after thoracolumbar spine surgery. J Korean Neurosurg Soc 2009;46:437-42.

3. Kim YJ, Bridwell KH, Lenke LG, Rhim S, Cheh G. Sagittal thoracic decompensation following long adult lumbar spinal instrumentation and fusion to L5 or S1: causes, prevalence, and risk factor analysis. Spine (Phila Pa 1976) 2006;31:2359-66.

4. Oh IS, Seo JY, Ha KY, Kim YC. Thoracic disc herniation of the adjacent segment with acutely progressing myelopathy. Asian Spine J 2010;4:52-6.

5. Watanabe K, Lenke LG, Bridwell KH, Kim YJ, Koester L, Hensley M. Proximal junctional vertebral fracture in adults after spinal deformity surgery using pedicle screw constructs: analysis of morphological features. Spine (Phila Pa 1976) 2010;35:138-45.

6. Yagi M, Akilah KB, Boachie-Adjei O. Incidence, risk factors and classification of proximal junctional kyphosis: surgical outcomes review of adult idiopathic scolio- sis. Spine (Phila Pa 1976) 2011;36:E60-8.

7. Becker S, Chavanne A, Spitaler R, et al. Assessment of different screw augmentation techniques and screw designs in osteoporotic spines. Eur Spine J 2008;17:14629.

8. Heini PF. The current treatment--a survey of osteoporotic fracture treatment. Osteoporotic spine fractures: the spine surgeon's perspective. Osteoporos Int 2005;16 Suppl 2:S85-92.

9. Kim JH, Kim SS, Suk SI. Incidence of proximal adjacent failure in adult lumbar deformity correction based on proximal fusion level. Asian Spine J 2007;1:19-26.

10. Kim YJ, Bridwell KH, Lenke LG, Rhim S, Kim YW. Is the T9, T11, or L1 the more reliable proximal level after adult lumbar or lumbosacral instrumented fusion to L5 or S1? Spine (Phila Pa 1976) 2007;32:2653-61.

11. DeWald CJ, Stanley T. Instrumentation-related complications of multilevel fusions for adult spinal deformity patients over age 65: surgical considerations and treatment options in patients with poor bone quality. Spine (Phila Pa 1976) 2006;31:S144-51.

12. Aydogan M, Ozturk C, Karatoprak O, Tezer M, Aksu $\mathrm{N}$, Hamzaoglu A. The pedicle screw fixation with vertebroplasty augmentation in the surgical treatment of the severe osteoporotic spines. J Spinal Disord Tech 2009;22:444-7.

13. Lattig F. Bone cement augmentation in the prevention of adjacent segment failure after multilevel adult deformity fusion. J Spinal Disord Tech 2009;22:439-43.

14. Voros G, Skolasky R, Robinson M, Kebaish K. Effect and outcome of prophylactic vertebroplasty in long spinal fusions on the incidence of proximal junctional kyphosis (PJK): minimum two year follow-up. Spine J 2007;7(5 Suppl):37S. 